\title{
A Novel High-Performance Linear Magnetic Gear
}

\author{
Kais Atallah Non-member (The University of Sheffield, k.atallah@sheffield.ac.uk) \\ Jiabin Wang Non-member (The University of Sheffield, j.b.wang@ @heffield.ac.uk) \\ Smail Mezani Non-member (INPL, smail.mezani@green.uhp-nancy.fr) \\ David Howe Non-member (The University of Sheffield, d.howe@ @sheffield.ac.uk)
}

Keywords: linear magnetic gear, linear machine, permanent magnets

The increasing demand for high force density actuators is currently being met almost exclusively by employing a leadscrew and nut to transform rotary motion to linear motion. However, an alternative approach is to employ a linear magnetic gear to increase the force capability of a conventional linear actuator. Therefore, the paper describes the design and performance of a linear magnetic gear having the tubular topology shown in Fig. 1 and whose parameters are given in Table 1.

Fig. 2 shows the variation of the maximum thrust force exerted on the low speed armature when a quasi-Halbach magnetization is employed for the high-speed armature. It can be seen that a force density in excess of $2 \mathrm{MN} / \mathrm{m}^{3}$ can be achieved. Fig. 3 shows how the force density of a magnetically geared actuation system comprising a linear magnetic gear and a linear permanent magnet brushless motor varies with the gear ratio, assuming the linear motor to have a force density of $0.3 \mathrm{MN} / \mathrm{m}^{3}$ and $0.6 \mathrm{MN} / \mathrm{m}^{3}$, these being typical values for naturally-cooled, and liquid-cooled permanent magnet linear brushless motors, respectively. As can be seen, the system force density for a gear ratio as low as $3.25: 1$ can be more than $100 \%$ greater than that of a naturally-cooled linear motor, for example.

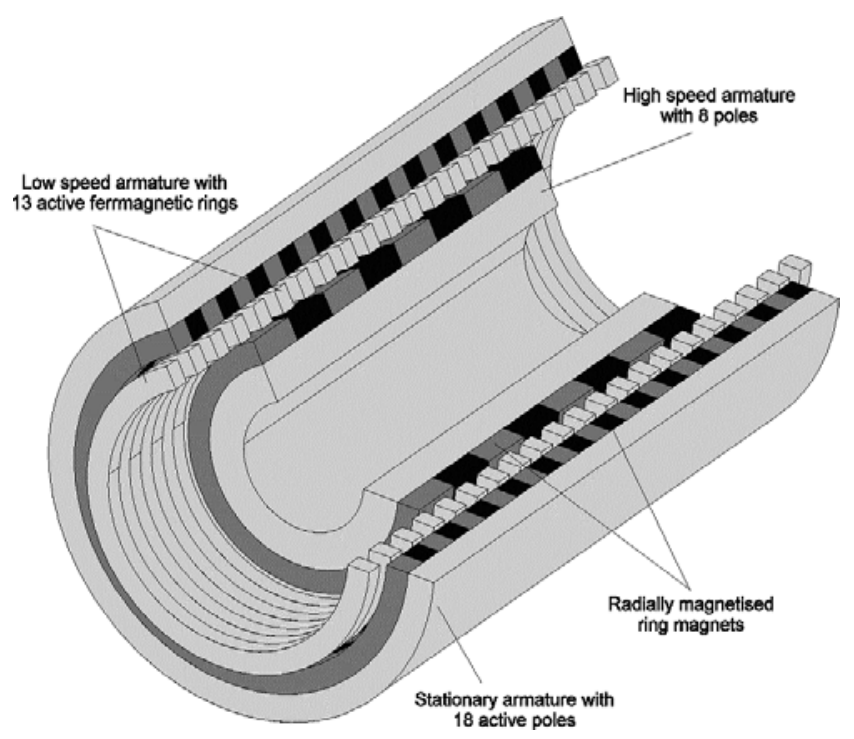

Fig. 1. Schematic of $3.25: 1$ linear magnetic gear
Table 1. Parameters of linear magnetic gear

\begin{tabular}{|l|c|}
\hline Gear ratio & $3.25: 1$ \\
\hline Diameter of stationary armature & $90 \mathrm{~mm}$ \\
\hline Active length of high speed armature & $100 \mathrm{~mm}$ \\
\hline Total length of stationary armature & $144.4 \mathrm{~mm}$ \\
\hline
\end{tabular}

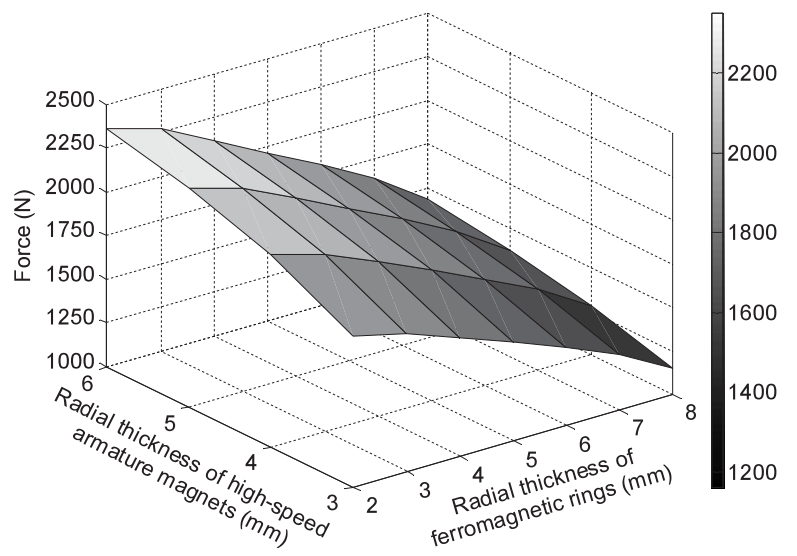

Fig. 2. Variation of maximum thrust force exerted on low speed armature with radial thickness of ferromagnetic rings and permanent magnets on high speed armature

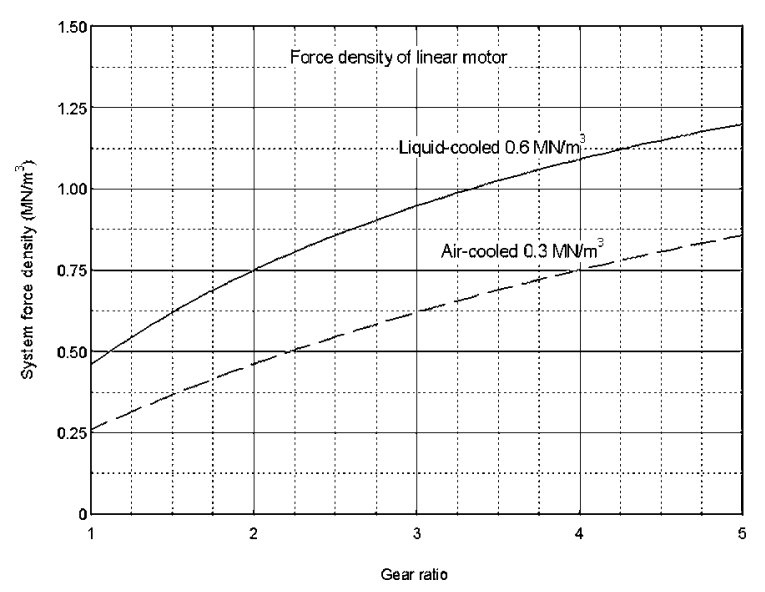

Fig. 3. Variation of system force density with gear ratio for a linear magnetic gear force density of $2 \mathrm{MN} / \mathrm{m}^{3}$ 


\title{
A Novel High-Performance Linear Magnetic Gear
}

\author{
Kais Atallah* Non-member \\ Jiabin Wang* Non-member \\ Smail Mezani* Non-member \\ David Howe* Non-member
}

Currently, the demand for high force density linear actuators is being met almost exclusively by employing a leadscrew and nut to transform rotary motion to linear motion. This paper describes an alternative technology, viz. a linear magnetic gear, which employs rare-earth magnets and which could be used in conjunction with a conventional linear actuator. Simulation studies have shown that such a gear has a highly competitive force transmission density.

Keywords: linear magnetic gear, linear machine, permanent magnets

\section{Introduction}

The increasing demand for high force density actuators is currently being met almost exclusively by employing a leadscrew and nut to transform rotary motion to linear motion. However, the efficiency of such helical force transformation systems can be relatively poor, particularly for low helix angles. In addition, wear and reliability can be significant issues, and lubrication may be required. An alternative approach is to employ a linear magnetic gear to increase the force capability of a conventional linear actuator, such as a linear brushless motor. To date, however, such an approach has received little, if any, attention.

The paper describes the design and performance of a linear magnetic gear having the tubular topology shown in Fig. 1, whose principle of operation is similar to that of the rotary and linear magnetic gears which were described in (1), (2) and (3). It is shown that, when rare-earth magnets are employed, such a linear magnetic gear can have a transmitted force density in excess of $2 \mathrm{MN} / \mathrm{m}^{3}$. Thus, when combined with a linear permanent magnet brushless motor, such a magnetic gear could offer significant advantages for many applications.

\section{Principle of Operation}

Fundamental to the operation of the magnetic gear is the modulation of the magnetic field produced by each of the concentric tubular permanent magnet armatures by the ferromagnetic rings (pole-pieces). These are disposed such that appropriate space harmonics result in the airgap magnetic field on each side of the pole-pieces, such that the magnetic field which results from one permanent magnet armature has the same number of poles as the associated permanent magnet armature. It can be shown, (2), that the number of polepairs in the space harmonic flux density distribution produced by either permanent magnet armature is given by:

\footnotetext{
* Department of Electronic and Electrical Engineering, The University of Sheffield

Mappin Street, Sheffield, S1 3JD, UK
}

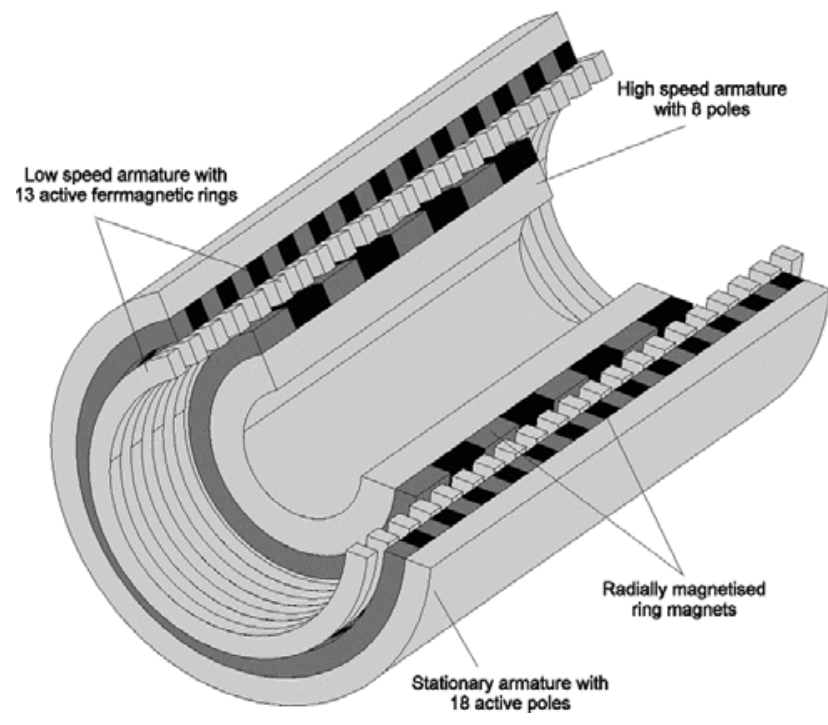

Fig. 1. Schematic of $3.25: 1$ linear magnetic gear

$$
\begin{aligned}
& p_{m, k}=\left|m p+k n_{s}\right| \cdots \ldots \ldots \ldots \ldots \ldots \ldots \ldots \ldots \\
& m=1,3,5, \ldots, \infty ; \quad k=0, \pm 1, \pm 2, \pm 3, \ldots, \pm \infty
\end{aligned}
$$

and that the linear velocity of the flux density space harmonics is given by:

$$
v_{m, k}=\frac{m p}{m p+k n_{s}} v_{r}+\frac{k n_{s}}{m p+k n_{s}} v_{s} \ldots \ldots \ldots \ldots \ldots
$$

where $p$ is the number of pole-pairs on the armature, $n_{s}$ is the number of ferromagnetic pole-pieces, and $v_{r}$ and $v_{s}$ are the linear velocity of the armature under consideration and the linear velocity of the pole-pieces, respectively. It will be noted that either the low speed permanent magnet armature or the ferromagnetic rings may be held stationary. However, in Fig. 1 it is assumed that the low speed armature is stationary.

From equation (2), it can be seen that the velocity of the space harmonics, which result due to the introduction of the ferromagnetic rings, viz. $k \neq 0$, is different to the velocity of the armature whose magnetic field they modulate. Therefore, in order to transmit thrust force at a different velocity, 
the number of active pole-pairs on the other permanent magnet armature must be equal to the number of pole-pairs in a space harmonic for which $k \neq 0$. Since the combination $m=1, k=-1$ results in the highest asynchronous space harmonic field, the number of pole-pairs of the other armature must be equal to $\left(n_{s}-p\right)$. The gear ratio is then given by:

$$
\mathrm{G}_{r}=\frac{n_{s}-p}{p}
$$

when the modulating ferromagnetic rings are held stationary, ie. $v_{s}=0$. On the other hand, if the low speed permanent magnet armature is held stationary, and the thrust force is transmitted to the ferromagnetic rings, the gear ratio becomes:

$$
\mathrm{G}_{r}=\frac{n_{s}}{p}
$$

This may be the preferred arrangement since it simplifies the mechanical design of the gear and enables a higher force to be transmitted, the increase being dependent on the gear ratio.

\section{Simulation Studies}

Table 1 gives the parameters which have been assumed for the linear magnetic gear shown in Fig. 1, which is equipped with radially magnetised sintered $\mathrm{NdFeB}$ permanent magnets having a remanence of $1.25 \mathrm{~T}$. 2-dimensional axis-symmetric magnetostatic finite element analysis has been employed to predict the flux density waveforms and the force transmission capability of the gear.

3.1 Flux Density Waveforms Fig. 2 shows the variation of the radial component of flux density due to the high speed, low pole number permanent magnet armature in the airgap adjacent to the stationary high pole number armature, whilst Fig. 3 shows the corresponding space harmonic spectrum. It can be seen that the presence of the ferromagnetic rings results in a number of asynchronous, viz. $k \neq 1$, space harmonics, the largest of which is the 9 pole-pair space harmonic, $(m=1, k=-1)$, which interacts with the 9 pole-pair

Table 1. Parameters of linear magnetic gear

\begin{tabular}{|l|c|}
\hline Number of pole-pairs on high speed armature & 4 \\
\hline Number of active pole-pairs on stationary armature & 9 \\
\hline Number of active ferromagnetic rings & 13 \\
\hline Gear ratio & $3.25: 1$ \\
\hline Diameter of stationary armature & $90 \mathrm{~mm}$ \\
\hline Diameter of high speed armature & $56 \mathrm{~mm}$ \\
\hline Active length of high speed armature & $100 \mathrm{~mm}$ \\
\hline Total length of stationary armature & $144.4 \mathrm{~mm}$ \\
\hline Air-gap length & $1 \mathrm{~mm}$ \\
\hline Radial thickness of stationary armature back-iron & $5 \mathrm{~mm}$ \\
\hline Radial thickness of high speed armature magnets & $L_{h}$ \\
\hline Radial thickness of ferromagnetic rings & $L_{f}$ \\
\hline
\end{tabular}

permanent magnets on the stationary armature to transmit a thrust force.

Similarly, Fig. 4 shows the variation of the radial component of flux density due to the permanent magnets mounted on the stationary armature in the airgap adjacent to the high speed armature, whilst Fig. 5 shows the corresponding space

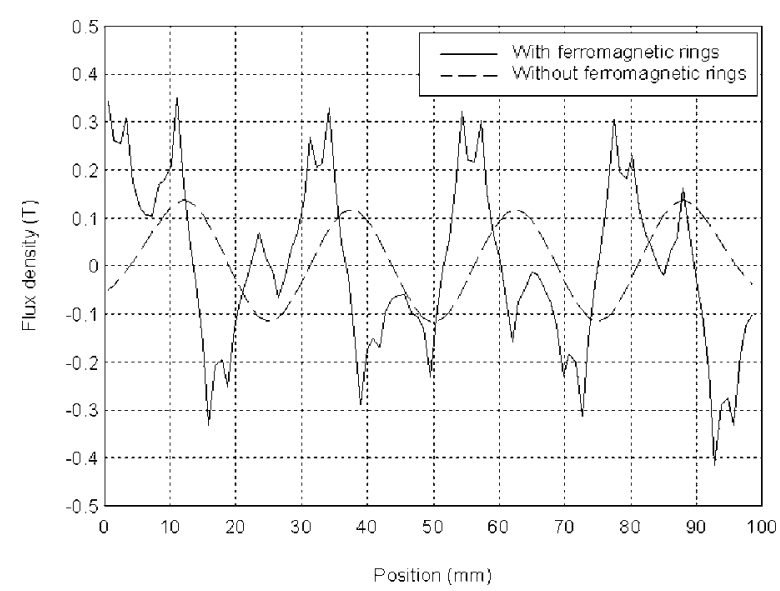

Fig. 2. Variation of radial flux density, due to high speed permanent magnet armature, in airgap adjacent to stationary armature

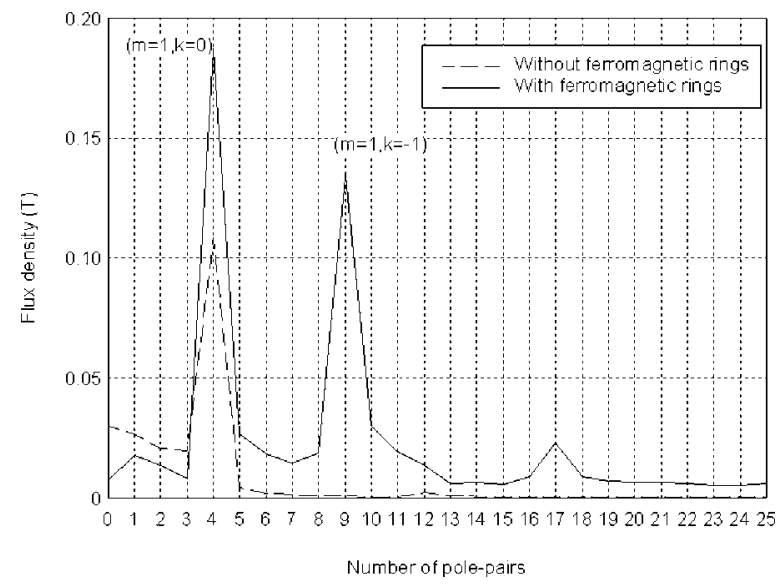

Fig. 3. Space harmonic spectrum of radial flux density, due to high speed armature, in airgap adjacent to stationary armature

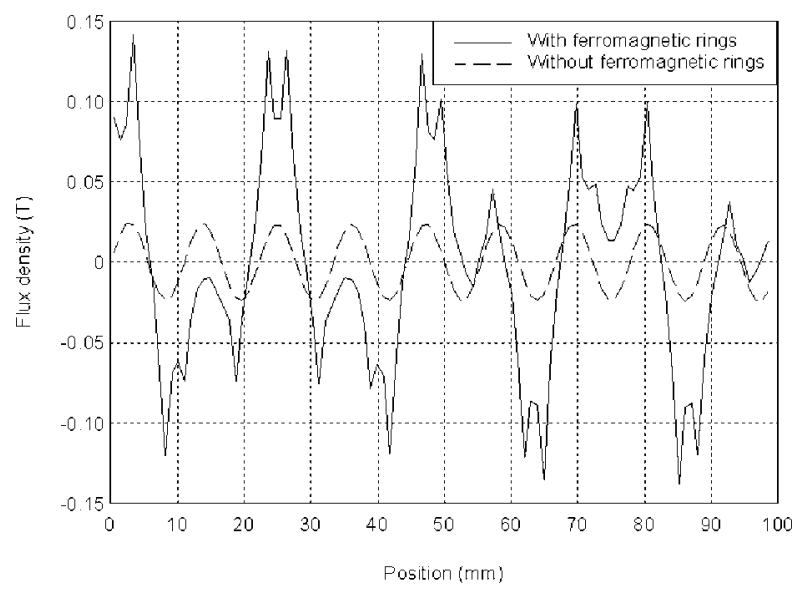

Fig. 4. Variation of radial flux density, due to stationary armature, in airgap adjacent to high speed armature 


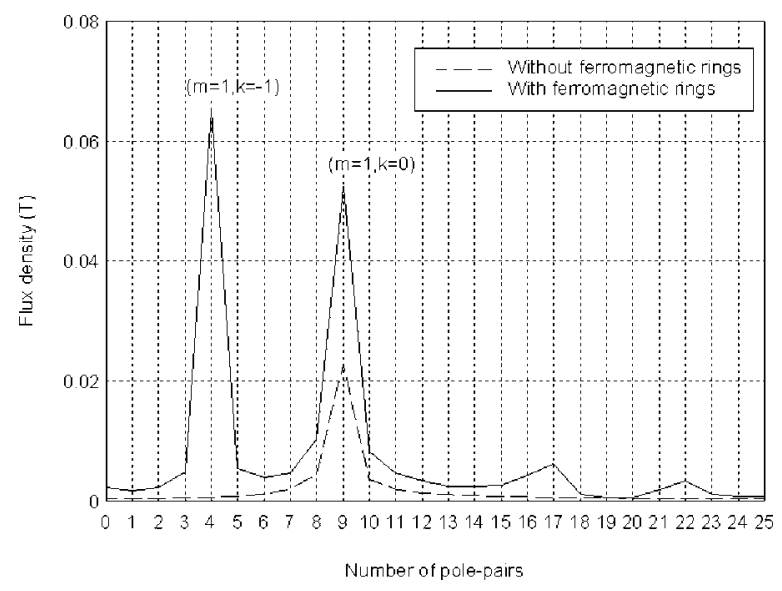

Fig. 5. Space harmonic spectrum of radial flux density, due to stationary armature, in airgap adjacent to high speed armature

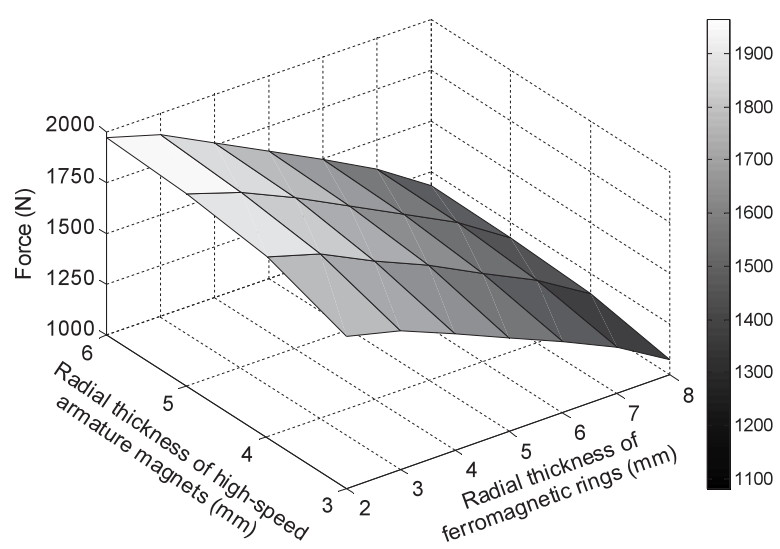

Fig. 6. Variation of maximum thrust force exerted on low speed armature with radial thickness of ferromagnetic rings and permanent magnets on high speed armature

harmonic spectrum. Again, it can be seen that the presence of the ferromagnetic rings results in a dominant 4 pole-pair asynchronous space harmonic, $(m=1, k=-1)$, which interacts with the 4 pole-pair permanent magnets on the high speed armature to transmit thrust force.

3.2 Thrust Force Fig. 6 shows the variation of the maximum thrust force exerted on the low speed armature with the radial thickness of both the high speed armature permanent magnets and the ferromagnetic rings when the magnets are radially magnetised, whilst Fig. 7 shows the variation of the thrust force which is exerted on the low speed and high speed armatures as they move linearly. It can be seen that the transmitted force remains fairly constant over a stroke of $\sim 20 \mathrm{~mm}$.

One way of increasing the force density is to employ quasiHalbach magnetized magnets on the high-speed, low pole number armature, such that each pole comprises a radially magnetised magnet and an axially magnetised magnet. By way of example, Fig. 8 shows the variation of the maximum thrust force exerted on the low speed armature with the ratio of the axial length of the radially magnetized ring magnets to the pole-pitch, $\alpha_{r}$, on the high speed armature, the

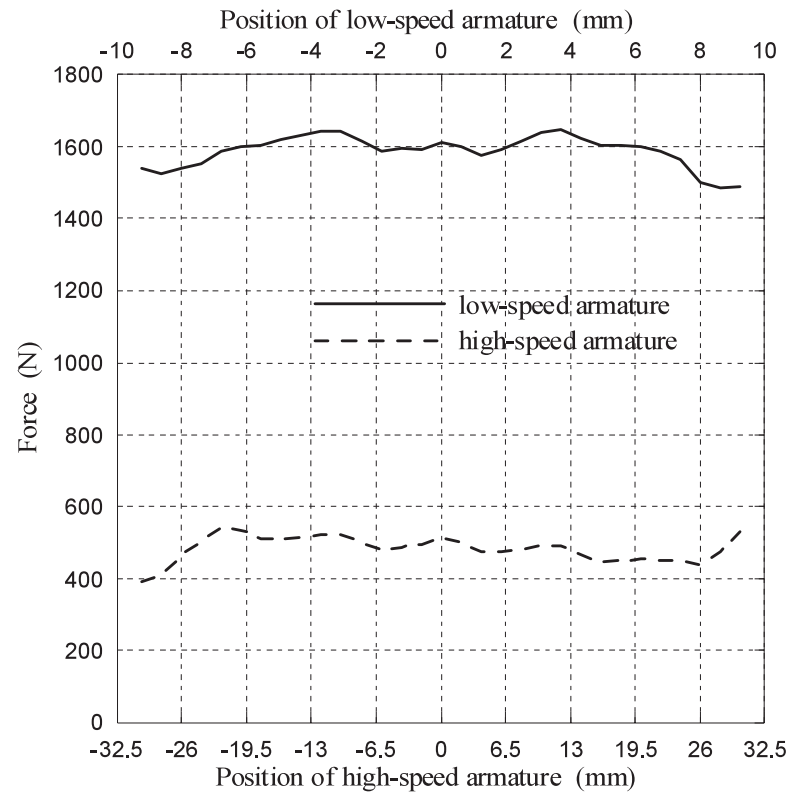

Fig. 7. Variation of maximum thrust force exerted on low speed and high speed armatures, $L_{h}=5 \mathrm{~mm}, L_{f}=$ $5 \mathrm{~mm}$

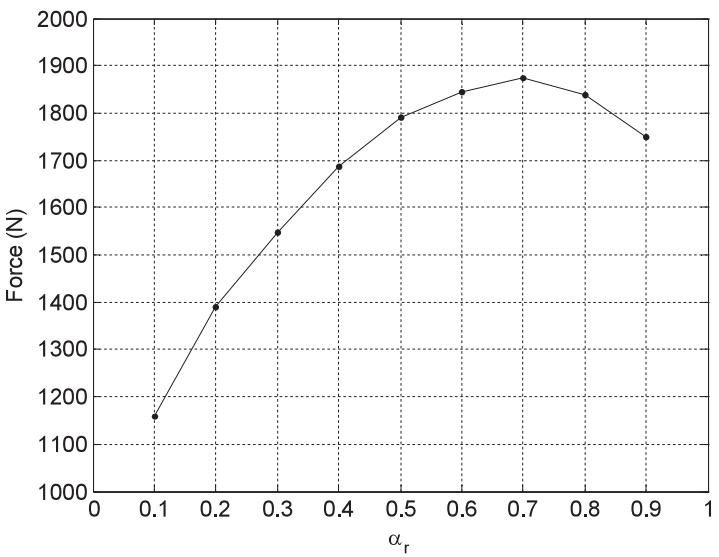

Fig. 8. Variation of maximum thrust force on low speed armature with $\alpha_{r} .\left(L_{h}=5 \mathrm{~mm}, L_{f}=5 \mathrm{~mm}\right)$

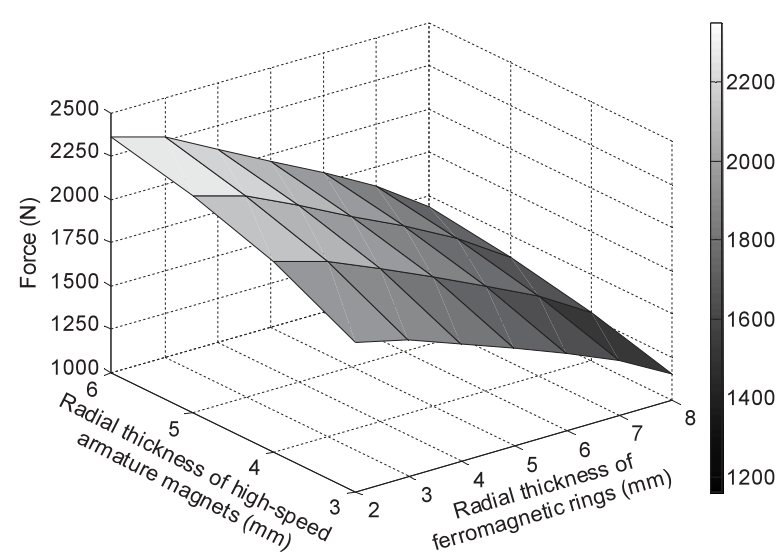

Fig. 9. Variation of maximum thrust force exerted on low speed armature with radial thickness of ferromagnetic rings and permanent magnets on high speed armature. (Halbach magnetization) 


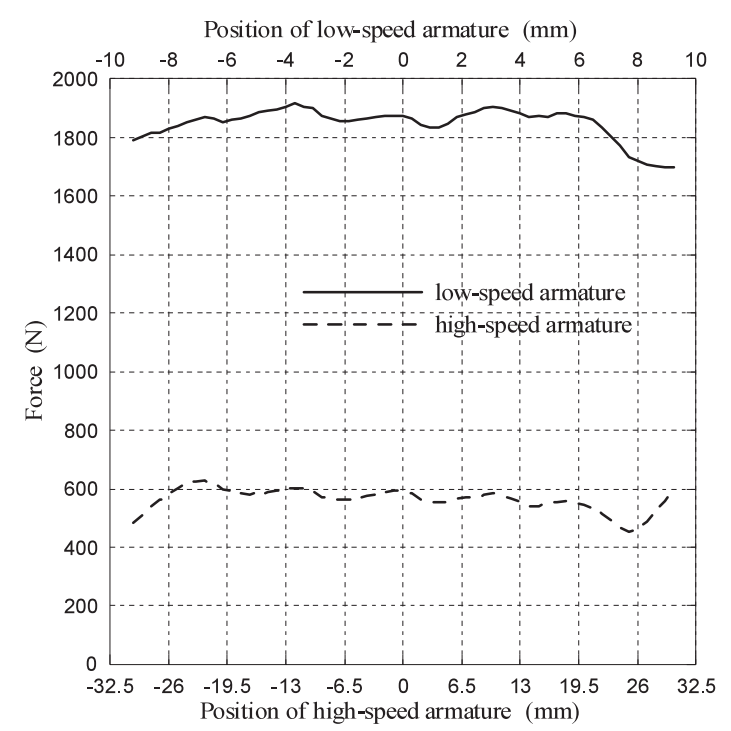

Fig. 10. Variation of maximum thrust force exerted on low speed and high speed armatures. (Halbach magnetization, $\alpha_{r}=0.7, L_{h}=5 \mathrm{~mm}, L_{f}=5 \mathrm{~mm}$ )

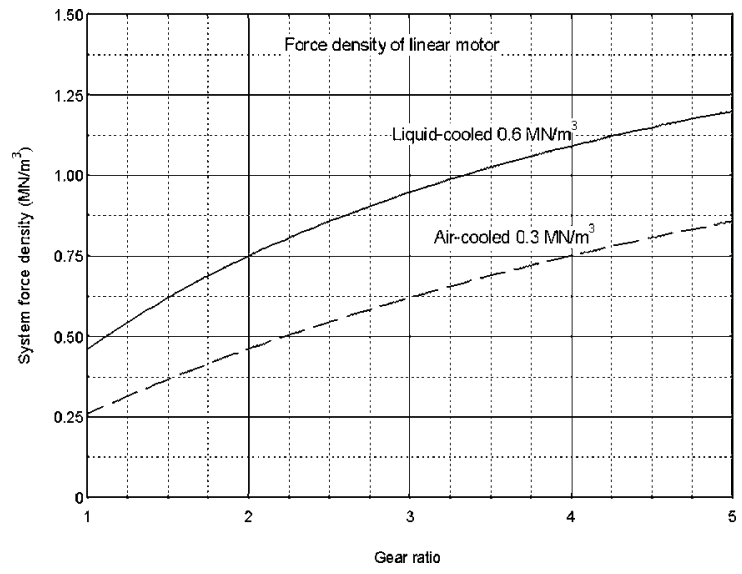

Fig. 11. Variation of system force density with gear ratio for a linear magnetic gear force density of $2 \mathrm{MN} / \mathrm{m}^{3}$.

magnets on the low speed armature being radially magnetised. It can be seen that the optimum value of $\alpha_{r}$ is 0.7 . Fig. 9 shows the variation of the maximum thrust force which is exerted on the low speed armature with the radial thickness of the high speed armature permanent magnets and the ferromagnetic rings, for $\alpha_{r}=0.7$. It can be seen that the quasi-Halbach magnetization results in a significantly higher thrust force than that which was developed when radially magnetized magnets were employed. Fig. 10 shows the variation of the thrust force which is exerted on the low speed and high speed armatures as they move linearly. As can be seen, a force density in excess of $2 \mathrm{MN} / \mathrm{m}^{3}$ can be achieved.

Fig. 11 shows how the force density of a magnetically geared actuation system comprising a linear magnetic gear and a linear permanent magnet brushless motor varies with the gear ratio, assuming the linear motor to have a force density of $0.3 \mathrm{MN} / \mathrm{m}^{3}$ and $0.6 \mathrm{MN} / \mathrm{m}^{3}$, these being typical values for naturally-cooled, and liquid-cooled permanent magnet linear brushless motors, respectively. As can be seen, the system force density for a gear ratio as low as $3.25: 1$ can be more than $100 \%$ greater than that of a naturally-cooled linear motor and more than $50 \%$ greater than that of a liquid-cooled linear motor. Therefore, the combination of a linear magnetic gear with a permanent magnet linear motor results in an actuation system, which has a significantly higher torque density than the motor alone.

\section{Conclusions}

The effects of design parameters on the thrust transmission capability of a high performance linear magnetic gear have been presented. It has been shown that, by employing a quasi-Halbach magnetized rare-earth magnets on the highspeed armature, a thrust force density of $\sim 2.0 \mathrm{MN} / \mathrm{m}^{3}$ can be achieved. It has also been shown that a high system force density for a combined linear magnetic gear and linear electrical machine can be achieved, even with a relatively low gear ratio.

(Manuscript received Nov. 25, 2005,

$$
\text { revised July 5, 2006) }
$$

\section{References}

( 1 ) K. Atallah and D. Howe: "A novel high-performance magnetic gear", IEEE Trans. on Magn., Vol.37, No.4, pp.2844-2846 (2001-4)

( 2 ) K. Atallah, S.D. Calverley, and D. Howe: "Design, analysis and realisation of a high-performance magnetic gear", IEE Proc. Electric Power Applic., Vol.151, pp.135-143 (2004)

( 3 ) K. Atallah, J. Wang, and D. Howe: "A high-performance linear magnetic gear", Journal of Applied Physics 97, 10N516 (2005)

Kais Atallah (Non-member) was born in Constantine, Algeria, in

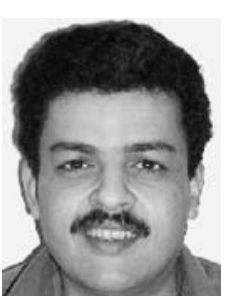
1964. He studied Electrical Power Engineering at Ecole Nationale Polytechnique in Algeria (Ingenieur d'Etat) and in 1993 he received the Ph.D. degree from the University of Sheffield, England. Currently he is a Senior Lecturer at the University of Sheffield, and he is also the Technical Director of Magnomatics Limited. From 1993 to 2000 he was a Postdoctoral Research Associate at the University of Sheffield. His research interests embrace high-performance permanent magnet drives for safety-critical applications, magnetic gearing and 'pseudo' direct drive electrical machines.

Jiabin Wang (Non-member) was born in Jiangsu Province, China, in

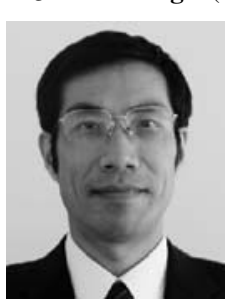
1958. He received the B.Eng. and M.Eng. degrees in electrical and electronic engineering from Jiangsu University of Science and Technology, Zhengjiang, China, in 1982 and 1986, respectively, and the Ph.D. degree in electrical and electronic engineering from the University of East London, London, U.K., in 1996. Currently, he is a Senior Lecturer at the University of Sheffield, Sheffield, U.K. From 1986 to 1991, he was with the Department of Electrical Engineering at Jiangsu University of Science and Technology, where he was appointed a Lecturer in 1987 and an Associated Professor in 1990. He was a Postdoctoral Research Associate at the University of Sheffield, Sheffield, U.K., from 1996 to 1997, and a Senior Lecturer at the University of East London from 1998 to 2001 . His research interests range from motion control to electromagnetic devices and their associated drives. 
Smail Mezani (Non-member) was born in Algiers, Algeria in 1974.

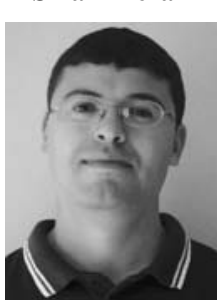
He studied Electrical Power Engineering at the University of Sciences and Technology in Algiers (Ingenieur d'Etat and Magister) and in 2004 he received a Ph.D. degree from Institut National Polytechnique de Lorraine, France. Currently he is a Lecturer at the University Henri Poincare, Nancy, France. From 2004 to 2005, he was a Postdoctoral Research Associate at the University of Sheffield, England, where he worked on magnetic gearing and 'pseudo' directdrive electrical machines. His research interests include the design of superconducting machines and loss computation in high temperature superconducting materials.
David Howe (Non-member) received the B.Tech. and M.Sc. degrees in electrical power engineering from the University of Bradford, Bradford, U.K., in 1966 and 1967, respectively, and the Ph.D. degree in electrical power engineering from the University of Southampton, Southampton, U.K. Currently, he is Professor of Electrical Engineering at the University of Sheffield, Sheffield, U.K., where he heads the Electrical Machines and Drives Research Group. He has held academic posts at Brunel University, London, U.K., and Southampton University, and spent a period in industry with NEI Parsons Ltd., Newcastle-Upon-Tyne, U.K., working on electromagnetic problems related to turbogenerators. His research activities span all facets of controlled electrical drive systems with particular emphasis on permanent-magnetexcited machines. Dr. Howe is a Fellow of the Institution of Electrical Engineers, and a Fellow of the UK Royal Academy of Engineering. 